Voix et Images

\title{
II.1 Périodiques littéraires et culturels du Québec depuis 1954
}

Volume 10, numéro 2, hiver 1985

La barre du jour / La nouvelle barre du jour

URI : https://id.erudit.org/iderudit/200499ar

DOI : https://doi.org/10.7202/200499ar

Aller au sommaire du numéro

Éditeur(s)

Université du Québec à Montréal

ISSN

0318-9201 (imprimé)

1705-933X (numérique)

Découvrir la revue

Citer cet article

(1985). II.1 Périodiques littéraires et culturels du Québec depuis 1954. Voix et Images, 10(2), 11-16. https://doi.org/10.7202/200499ar d'utilisation que vous pouvez consulter en ligne.

https://apropos.erudit.org/fr/usagers/politique-dutilisation/ 


\section{TABLEAUX}

\section{II.1 Périodiques littéraires et culturels du Québec depuis 1954}

Le plus périodique littéraire du Québec étant actuellement, et depuis quelque trente ans, les Écrits du Canada français, il n'est pas inutile de poser chronologiquement, parallelement à la suite ininterrompue de ses numéros et d'une manière qui ne se prétend nullement exhaustive, le surgissement des autres périodiques littéraires et culturels du Québec. On trouvera ici l'année de fondation (avec, selon le cas, le mois ou le trimestre) et, chaque fois que nous la connaissons, l'année de disparition. Les quelques périodiques fondés avant 1954 mais disparus après 1954 ou encore publiés apparaissent à leur place, au tout début de cette liste. Tous les périodiques sont publiés à Montréal, sauf indication contraire. Exceptionnellement, figurent ici des périodiques non québécois mais qui font une bonne place à la littérature québécoise.

Près de 140 périodiques sont nés entre 1954 et 1984, dont 60 environ sont aujourd'hui disparus.

1919

Le Quartier latin (janvier 1919 - novembre 1970)

1920

The Canadian Forum (1920), Toronto

1931

The University of Toronto Quarterly (1931), Toronto

1933

L'Action nationale (1933)

1936

Les Carnets victoriens (1936), Joliette

1940

Culture (mars 1940 - mars 1971) 
12 VOIX ET IMAGES, VOL. X, NO 2 (HIVER 85)

1941

Relations (1941)

Amérique française (novembre 1941 - 1955)

1946

Lectures (septembre 1946 - juin 1966)

1950

Cité libre (1950 - 1966)

1947

Revue d'histoire de l'Amérique française (juin 1947)

1951

La Nouvelle Revue canadienne (1951 - 1956), Ottawa

Arts et Pensée (1951 - 1955)

1953

Emourie (mars 1953-hiver 1965), Québec

1954

Les Écrits du Canada français (août 1954)

1955

Séquences (1955)

1956

Les Cahiers de l'Académie canadienne-française (1956)

Vie des arts (1956)

Le Message des poètes (novembre 1956-janvier/mars 1965) - voir Poésie (1966)

1959

Liberté (janvier/février 1959)

Situations (janvier 1959-juillet 1962)

La Revue socialiste (printemps 1959)

Canadian Literature (été 1959), Vancouver

1960

Actualité (janvier 1960)

Recherches sociographiques (janvier/mars 1960)

Châtelaine (octobre 1960)

Revue de l'Université de Sherbrooke (novembre 1960-juin 1965), Sherbrooke

1961

La Poubelle littéraire (janvier 1961)

Livres et Auteurs canadiens (1961-1968) - voir 1969

1962

Maintenant (janvier 1962-décembre 1972)

Incidences (novembre 1962-avril 1969), Ottawa - voir Co-Incidences (1971) 
1963

L'Indépendantiste (mars 1963-printemps 1968)

L'Odyssée (janvier 1963)

Parti pris (octobre 1963-octobre 1968)

Jeunesse littéraires du Canada français (décembre 1963-mars 1967

Lettres et Écritures (décembre 1963-novembre 1968)

1964

Québec 64 (mai 1964-mai 1972)

Dimensions Digeste Éclair (1964-1972)

1965

Passe-partout (janvier 1965-novembre/décembre 1965), Saint-Constant

Vient de paraître (janvier 1965-septembre 1973)

La Barre du Jour (février 1965-mai/août 1977) - voir 1977

Etudes françaises (février 1965)

La Tourmente (1965-1967), Québec

1966

Cahiers de Sainte-Marie (mai 1966-avril 1969) - voir Voix et Images du Pays (1970)

Poésie (hiver 1966-été 1979), Québec

Théatre vivant (1966-1968)

Culture vivante (1966-septembre 1973)

Québec-Amérique (1966)

Emergences (1966-1968), Trois-Rivières

1967

Quoi (janvier/février 1967-printemps/été 1967)

Ratures (mars 1967), Québec

Forces (hiver 1967)

Sexus (aout-septembre 1967-1968)

1968

Études littéraires (avril 1968), Québec

La Revue de l'Université de Moncton (mai 1968-septembre 1973), Moncton

- voir Si que (1974)

Les Herbes rouges (octobre 1968)

Délirium très minces (hiver 1968-1969)

Asticou (1968)

1969

Illustrations babéliennes (avril 1969-1973)

Socioloie et Sociétés (mai 1969).

Littérature canadienne/Canadian Literature (été 1969-hiver 1973),

Vancouver

Cahiers François-Xavier Garneau (septembre 1969), Québec

Carnets des Auteurs réunis (octobre 1969-1971), Windsor

Ellipse (automne 1969), Sherbrooke

Orphée (4e trimestre 1969)

Livres et Auteurs québécois (1969) 
1970

Ether (janvier 1970-mars 1971), Longueuil

L'Illettré (janvier 1970-mai 1971), Terrebonne

Nos livres (janvier 1970)

Critère (février 1971)

Inédits (début 1971), Québec

Voix et Images du Pays (2e trimestre 1970-1975) - voir 1975

Mainmise (octobre 1970-été 1978)

Québec français (journal) (novembre 1970), Québec — voir 1974

Présence francophone (automne 1970), Sherbrooke

Bulletin du Centre de recherche en civilisation canadienne-française (décembre 1970), Ottawa

Protée (décembre 1970), Chicoutimi

Point de mire (1970-mai 1972)

1971

Co-Incidences (mars 1971-novembre/décembre 1976), Ottawa - voir Incidences (1977)

Cinéma-Québec (mai 1971-novembre/décembre 1973)

Nord (octobre 1971-novembre 1977), Québec

Presqu'Amérique (octobre 1971-février 1973)

Recherches amérindiennes au Québec (1971), Québec

1972

Le Chien d'or/The Golden Dog (janvier 1972-février 1974)

Etudes françaises dans le monde (novembre 1972)

Osiris (novembre 1972), Deerfield (Massachusetts)

Stratégie (hiver 1972-automne 1977)

Les Cahiers de Cap-Rouge (décembre 1972), Cap-Rouge

Hobo-Québec (décembre 1972-hiver 1981)

1973

Brèches (printemps 1973-1977)

Cul $Q$ (automne 1973-1977)

1974

Québec français (revue) (janvier 1974), Québec

Le Québec littéraire (1er trimestre 1974-1976)

Le Temps fou (février/mars 1974-septembre 1983)

Aspects (mai 1974-janvier 1976), Québec

Si que (mai 1974), Moncton

L'Ecran (juin 1974)

Requiem (septembre 1974-juin/juillet 1979) - voir Solaris (1979)

Champs d'application (hiver 1974-hiver 1975), Trois-Rivières

Résistance (1974), Sherbrooke

1975

Chroniques (janvier 1975-hiver 1978)

Lettres québécoises (mars 1975)

Biblio (été 1975) 
Voix et Images (septembre 1975)

Dérives (septembre/octobre 1975)

Parachute (octobre 1975)

Études canadiennes (1975), Bordeaux/Lille (France)

1976

L'Échancrier (janvier-septembre 1976), Sherbrooke

Jeu (janvier 1976)

Les Têtes de pioche (mars 1976-juin 1979)

Estuaire (mai 1976), Québec

Propos d'art (octobre 1976-hiver 1980/1981)

Possibles (automne 1976)

Trac (décembre 1976)

Atelier de Production littéraire de la Mauricie (1976-1982), Trois-Rivières

- voir 1984

1977

Incidences (janvier 1977-septembre/décembre 1982), Ottawa

Moebius (2e trimestre 1977)

La Nouvelle Barre du Jour (septembre 1977)

La Grande réplique (octobre 1977-1981) - voir Pratiques théâtrales (1981)

1978

Mille plumes (janvier 1978)

Grimoire (février 1978-avril 1983), Sherbrooke

Intervention (mai 1978-1984), Québec - voir Inter (1984)

Association des Editeurs de périodiques culturels québécois - (été 1978)

Cahiers de la femme/Canadian Women Studies (automne 1978-1981)

L'Écritoire (1978-1979)

Focus (1978-1981), Saguenay/Lac Saint-Jean

1979

Les Cahiers du Hibou (avril 1979-1980), Sherbrooke

Ecriture française dans le monde (mai 1979), Sherbrooke

Cahiers des arts visuels (printemps 1979)

Spirale (septembre 1979)

Imagine (septembre/novembre 1979)

Copie zéro (1979)

La Gazette des Femmes (1979)

Solaris (1979)

Trajectoires (1979), Dollard-des-Ormeaux

1980

La Vie en rose (mars 1980)

Revue d'Histoire littéraire du Québec et du Canada français (2e trimestre 1980)

Le Bulletin Pantoute (avril 1980-janvier/février 1981) - voir Nuit blanche (1982)

Les Cahiers d'art et lettres du Québec (1980)

Hom-Info (1980)

L'Insolite Sherbrooke (hiver 1980), Sherbrooke 
16 VOIX ET IMAGES, VOL. X, NO 2 (HIVER 85)

\section{1}

Urgences (avril 1981), Rimouski

Sonances (octobre 1981)

Pratiques theatrales (automne 1981)

Cahiers des arts visuels au Québec (1981)

1982

Arcade (printemps 1982)

Nuit blanche (aoât 1982), Québec

Résistances (1982), Saguenay/Lac Saint-Jean

1983

Lèvres urbaines (2e trimestre 1983)

Passages (automne 1983), Sherbrooke

Le Sabord (octobre/novembre 1983), Trois-Rivières

1984

Esquisses (1er trimestre 1984), Sorel

Dixit-0l (hiver 1984)

Nouaison (printemps 1984), Trois-Rivières

Atelier de Production littéraire des Forges (2e trimestre 1984), TroisRivières

Les Deux Rives (juin 1984), Paris (France)

Emergence Poésie (juin 1984)

Inter (octobre 1984)

Carfax (1984), Hull 